\title{
Erratum to: Neuroprotective Effect of the Endogenous Amine 1MeTIQ in an Animal Model of Parkinson's Disease
}

\author{
Agnieszka Wasik $^{1}$ - Irena Romańska ${ }^{1} \cdot$ Jerzy Michaluk $^{1} \cdot$ Agnieszka Zelek-Molik $^{2}$. \\ Irena Nalepa ${ }^{2} \cdot$ Lucyna Antkiewicz-Michaluk $^{1}$
}

Published online: 22 April 2016

(c) Springer Science+Business Media New York 2016

\section{Erratum to: Neurotox Res (2016) 29:351-363 DOI 10.1007/s12640-015-9556-6}

In the original publication, the acknowledgment section was published incorrectly. The correct acknowledgment is given below:
Acknowledgments The authors would like to thank Maria Kafel and Krzysztof Michalski for technical assistance. This study was financially supported through a grant from the National Science Centre, Grant No. DEC-2012/ 07/B/NZ7/01149, and statutory funds from the Institute of Pharmacology, Polish Academy of Sciences, Krakow, Poland.

The online version of the original article can be found under doi:10.1007/s12640-015-9556-6.

Agnieszka Wasik

wasik@if-pan.krakow.pl

1 Department of Neurochemistry, Institute of Pharmacology,

Polish Academy of Sciences, 12 Smetna Street,

31-343 Krakow, Poland

2 Department of Biochemistry, Institute of Pharmacology,

Polish Academy of Sciences, 12 Smetna Street,

31-343 Krakow, Poland 